\title{
Valoración de activos biológicos aplicados en el cultivo de Aguacate Hass, a través de la evaluación por expansión bajo la metodología de opciones reales
}

\author{
Valuation of biological assets applied in the cultivation of avocado hass, through the \\ evaluation by expansion under the methodology of real options
}

\section{Hector Fabio Lopez Castaño ${ }^{1,}$ Ricardo Alonso Colmenares Florez ${ }^{2}$, Denis Augusto Lara Papamija ${ }^{3}$}

\footnotetext{
${ }^{1}$ Ingeniero Agrónomo, Magister en Administración Financiera, PMP. Docente investigador de la Escuela de Ciencias Agrícolas, Pecuarias y del Medio Ambiente, ECAPMA de la UNAD. ORCID https://orcid.org/0000-0001-5541-1577

${ }^{2}$ Contador Público, MBA en Dirección y Administración de Empresas. doctor en administración empresarial, especialista en revisoría fiscal y auditoria forense, docente investigador. ORCID https://orcid.org/0000-0003-3215$\underline{8868}$

${ }^{3}$ Contador Público, Especialista en Finanzas, Magister en Administración financiera, Especialista en revisoría Fiscal, especialista en administración de la informática educativa, docente Investigador grupo ciencias de la gestión. ORCID https://orcid.org/0000-0003-4302-1146
}

DOI: $\underline{\text { https://doi.org/10.46589/rdiasf.vi35.408 }}$

Recibido 13 de febrero 2021.

Aceptado 6 de junio 2021

Publicado 30 de junio de 2021

\section{Resumen}

El presente trabajo permite abordar la contabilización de los activos biológicos a valor razonable como herramienta útil y generadora de valor para una asociación de productores de aguacate hass, desde el inicio de la producción hasta la culminación del ejercicio, así mismo permitirá la valoración de un proyecto de inversión el cual contiene la expansión y contracción de dicho proyecto el cual se valorará por el sistema tradicional de valor presente neto y así mismo por la metodología de opciones reales, incluyendo los árboles binomiales, donde se puede concluir que la contabilización a valor razonable del activo real, puede generar valor en su rentabilidad de acuerdo al estándar internacional en cualquier tiempo durante la producción, de igual manera se 
devela que el proyecto de inversión valorado por el sistema tradicional no es viable, y al valorar el proyecto bajo la metodología por opciones reales genera flexibilidad la cual permite generar valor y que el proyecto sea rentable para la asociación.

Palabras clave: Activos biológicos, opciones reales, opción compuesta, Niif, Nic.

\begin{abstract}
This work allows addressing the accounting of biological assets at fair value as a useful and value-generating tool for an association of Hass avocado producers, from the beginning of production to the end of the year, as well as allowing the valuation of a project of investment which contains the expansion and contraction of said project which will be valued by the traditional system of net present value and also by the methodology of real options, including binomial trees, where it can be concluded that the accounting at fair value of the real asset, can generate value in its profitability according to the international standard at any time during production, in the same way it is revealed that the investment project valued by the traditional system is not viable, and when valuing the project under the option methodology real generates flexibility which allows generating value and that the project is profitable for the association.
\end{abstract}

Keywords: Biological assets, real options, compound option, Niif, Nic.

\title{
1. Introducción
}

En el ámbito financiero de valoración de activos a nivel general existe una amplia ambigüedad, sobre las metodologías de medición a valor razonable de los activos biológicos bajo la norma internacional de información financiera y la norma internacional de contabilidad, el universo temático de investigación y las corrientes de investigación empírica o aplicada son muy incipientes. En este sentido el panorama dificulta la configuración de empresas, sociedades o comunidades campesinas sobre la contextualización como actores sociales. 
La norma internacional en el proceso de convergencia de normas locales a NIIF, enuncia que las diferentes empresas, en especial las que pertenecen al grupo 1, denominado de las NIIF plenas, deben dar reconocimiento de acuerdo con valor razonable a sus diferentes activos, tema que trata de manera exclusiva la NIIF 13, que además de abordar lo referente a valoración de los activos, también lo hace sobre los pasivos al realizar la transferencia entre los actores del mercado, así mismo la NIC 41 permite abordar la agricultura como activos biológicos, destacando la importancia que tiene para el sector primario de la economía y da directrices para el tratamiento contable y la información que se revela con relación a este renglón, lo que permitirá manejar niveles de competitividad.

De igual forma en la valoración de proyectos de inversión se hace necesario tener en cuenta tres escenarios, entre ellos la reversibilidad o irreversibilidad de la inversión ante un resultado adverso, la mejor opción para proceder a invertir y la incertidumbre, teniendo en cuenta estos escenarios podemos destacar que la valoración de activos de inversión por el método VPN deja de lado la flexibilidad la que se puede incurrir durante el procesos por tanto se hace necesario incluir en dicha valoración, métodos alternativos como son la valoración por opciones reales. Para este caso en donde se pretende analizar un proyecto de inversión para expansión en hectáreas de producción de aguacate Hass, se hará un comparativo en la valoración de activos biológicos bajo la teoría VPN y las opciones reales, generando el espacio para determinar la flexibilidad del proyecto de inversión.

Para el tema objeto de estudio se analizara la producción de aguacate hass como activo biológico, teniendo en cuenta que a la fecha las exportaciones del fruto se ha incrementado en promedio de 26 millones de dólares con respecto al año 2018, siendo la exportación del 2019 de 89 millones de dólares, lo que permite visualizar que la internacionalización tiene un efecto positivo en la oferta colombiana, que a través del tiempo puede mejorar la competitividad y la adopción de nuevas prácticas que generan valor agregado a los productores mejorando el ingreso y calidad de vida.

En este sentido el presente trabajo permite analizar mediante la medición contable a valor razonable y una metodología de comparación entre los sistemas tradicionales de valoración 
financiera VPN y uno más reciente que tiene en cuenta los riesgos implícitos del sector agrícola, tales como las opciones reales, dando alcance al tema de investigación, así mismo se permite realizar la revisión bibliográfica basada en referentes y estudios pertinentes con respecto a contabilización y valoración de activos biológicos.

\section{2- Estudio problema}

Mediante la ley 1314 de 2009, y el decreto 2420 de 2015 el estado colombiano adoptó por convergencia, y reguló los principios y las normas internacionales de contabilidad e información financiera y de aseguramiento de la información, en esta ley indicó las competencias de las organizaciones y la ruta para su expedición, así mismo se determinaron las entidades del gobierno responsables de regular su cumplimiento. Tomando como referencia la ley 1314 de 2009, la Presidencia de la República en el año 2012 expidió los decretos 2784 de 2012, 2706 y 3022 de 2013, en los cuales se refiere expresamente al proceso de adopción, así como los requisitos, el cronograma y el grupo de NIIF, en las cuales quedaron inmersas las empresas para preparar sus estados financieros (Tohmatsu, D. T, 2013).

Es así como la clasificación de las normas internacionales de información financiera en el caso colombiano, se pueden clasificar bajo niif plenas; las cuales se aplican de una manera completa a las organizaciones y las niif para pymes las cuales no se aplican de manera completa omitiendo algunos temas específicos, de acuerdo a sus necesidades y su aplicación se da desde los mismos principios de contabilidad, de igual forma desde el punto de vista objetivo de la contabilidad se aprueba que las empresas se puedan clasificar en diferentes grupos, con diferentes criterios. Bajo los grupos a los cuales hace relación la norma para la convergencia de las Normas Internacionales de Información Financiera, se fundamenta en el tamaño de las empresas, el número de trabajadores, ingresos, activos cuya caracterización se evidencia a continuación:

Grupo uno: reglamentado por el decreto 2784 de 2012, pertenecen los emisores de valores o empresas que cotizan en bolsa, entidades de interés público las cuales están obligadas a 
hacer rendición de cuentas, su descripción es grandes empresas las cuales tienen activos totales superiores a treinta mil salarios mínimos mensuales legales vigentes (SMMLV), o que cuenten con una planta de personal de más de 200 trabajadores, así como organizaciones que realicen $50 \%$ de importaciones o exportaciones del total de sus operaciones, además si es matriz o subordinada de empresa nacional o extranjera.

Grupo dos: Reglamentado por el decreto 3022 de 2013, están incluidas las empresas de tamaño grande o mediano, que no cotizan en bolsa, ni están obligadas a rendir cuentas, adicionalmente que preparen sus estados financieros sobre el estándar para Pymes.

Grupo tres reglamentado por el decreto 2706, de 2012, pertenecen las pequeñas microempresas a quien expresamente se les autoriza la emisión de sus estados financieros y las respectivas revelaciones de forma abreviada, dentro de las condiciones relevantes se encuentran el tener activos inferiores a 500 salarios mínimos legales vigentes, ingresos inferiores a 6.000 salarios mínimos legales vigentes y tener una planta de personal por debajo de 10 trabajadores.

Teniendo en cuenta el proceso de convergencia de las NIIF y en el tratamiento que se ha venido llevando a cabo en las empresas agrícolas con respecto a sus activos biológicos y su contabilización, especialmente en el del cultivo de Aguacate hass, en su valoración desde su siembra, etapa productiva, hasta la etapa de soqueo, se evidencia oportunidades de mejora sustanciales, acompañado de este proceso se hace necesario implementar metodologías comparativas de valoración entre el método tradicional VPN Y nuevos métodos de valoración como las opciones reales las cuales van a permitir evaluar los flujos de ingreso, de diferentes escenarios a diferentes nivel de ventas y de productividad, constituyéndose en alternativa eficaz para la toma de decisiones en el marco de la gestión integral de riesgo.

Con base en lo que representa el cultivo de aguacate hass y el reconocimiento actual como producto de exportación y la aplicación de su adecuado tratamiento según las normas contables actuales de contabilidad y la valoración financiera, le permitirá al agricultor tomar mejores decisiones a la hora de invertir, vender, ampliar la inversión del cultivo o abandonar la inversión, surge un gran interrogante. 
¿Cómo valorar en forma adecuada un activo biológico de Aguacate hass bajo los estándares internacionales de contabilidad y metodología de valoración bajo el enfoque de opciones reales?

\section{Objetivos}

\subsection{General}

Definir la metodología de valoración para el cultivo de Aguacate hass bajo el estándar internacional, con el enfoque en opciones reales.

\subsection{Específicos}

- 1.Aplicar la metodología de valoración en la determinación del valor razonable del cultivo de aguacate Hass, bajo normas internacionales de información financiera.

- 2- Diseñar la metodología para la valoración del cultivo de Aguacate hass, con base en Opciones reales.

- 3. Aplicar la metodología, comparando el método tradicional VPN y opciones reales en el cultivo de aguacate.

\section{Marco de referencia conceptual}

la contabilidad ha presentado dificultades al hacer la contabilización de los activos biológicos en Colombia, por lo tanto, se hace necesario apegarse a los estándares internacionales de contabilidad para definir métodos de valuación a valor razonable establecidos en los estándares internacionales de información financiera.

Según Palavecinos, B. S. (2011), valor razonable, es un concepto relevante que la International Accounting Standards Board (IASB) lo incluyó como un modelo de valoración en las Normas Internacionales de Información Financiera, asimilando la medición del valor razonable a la medición del mercado y no en la medición de una entidad específica lo que permite 
dejar abierta la posibilidad a los participantes de dicho mercado sean quienes asuman el valor del activo.

Al respecto, (Carrillo, 2015), determina que el valor razonable como el precio que se recibe por enajenar un activo o el valor pagado para transferir un pasivo, teniendo en cuenta las siguientes características, que el activo o pasivo debe ser consecuente con su unidad de cuenta, así mismo para los activos no financieros los supuestos de valuación deben ser conforme a su consistencia y su uso.

De otra parte García, López y Cerón (2015), determinan la importancia de la actividad agrícola en la conservación ambiental, la disminución de la pobreza y el hambre, y la seguridad alimentaria, lo anterior devela la intervención notoria en los mercados globales del sector agrícola, lo cual obliga a las organizaciones y asociaciones a presentar sus estados financieros de forma clara y transparente acogiendo las Normas Internacionales de Información Financiera, teniendo en cuenta la aplicación de la NIC 41 en Colombia.

Así mismo, relacionando los elementos del costo, Uribe (2011), demuestra que es un factor importante para la contabilidad y la valoración por métodos tradicionales o para sistemas modernos de valuación, en donde menciona su caracterización e importancia describiendo, los diferentes usos y discriminación dentro los cuales encontramos, las materias primas, la mano de obra y los costos indirectos de fabricación.

\section{Valoración de proyectos.}

Tradicionalmente, el análisis del flujo de caja descontado junto con el valor actual neto, han sido las principales herramientas de apoyo a la hora de tomar decisiones de inversión por parte de los profesionales en finanzas; sin embargo, algunos autores como Myers (1984) y Hull (2018), indican las desventajas de esta técnica de evaluación, pues no contempla la flexibilidad propia que suelen expresar los flujos de caja de las empresas.

De esta manera, el indicador presume que solo se presentarán flujos negativos al inicio de la actividad y continuamente flujos positivos en otros años, dejando de lado la posibilidad común que se presenten años negativos alternados con positivos en los períodos siguientes, en 
concordancia con lo expuesto por Dixit \& Pindyck (1994), en el sentido de la poca flexibilidad de este indicador financiero

\section{Valoración por opciones reales.}

En consecuencia, Myers (1977) introduce el concepto de opciones reales como una solución para encontrar el valor total los proyectos, incluyendo la flexibilidad en cada uno de sus etapas. Posteriormente, Myers (1984) establece el término de opciones reales, vinculándolo con el activo subyacente con un activo real

Como un método alternativo de valoración ante las limitaciones de la metodología DCF, surge la teoría de real options approach (ROA, «opciones reales») (Myers y Turnbull, 1977), enfoque que complementa el tradicional DCF y que permite incluir la flexibilidad del negocio mediante decisiones, como por ejemplo, diferir, expandir o abandonar, entre otras.

En esta metodología se resalta el empleo de algunos aspectos relacionados de modo directo con las patentes, como son la incertidumbre y la volatilidad sobre los ingresos, costos e inversiones, y la contingencia, entre otros (Reyck, Degraeve y Gustafsson, 2004). En otras palabras, esta metodología permite incluir una mayor flexibilidad en la toma de decisiones de inversión y de renovación, como también para realizar solicitudes internacionales de patentabilidad o de abandonar (Smith y Nau, 1995).

Bajo este contexto, una opción se puede definir como un método de valoración de activos reales que presentan un componente de flexibilidad, como lo es la inversión en investigación y desarrollo.

El término de opciones reales fue establecido por Myers en 1977, para hacer referencia a la aplicación de la teoría de opciones en la valoración de bienes no financieros. De ahí la diferencia de parámetros que influyen a la hora de valorar una opción financiera y una real. 
Tabla 1. Parámetros opciones financieras y opciones reales

\begin{tabular}{|c|c|}
\hline Opción Financiera & Opción Real \\
\hline Precio del activo Subyacente & $\begin{array}{l}\text { Valores esperados de los flujos de } \\
\text { caja }\end{array}$ \\
\hline Precio de ejercicio & Costo de inversión \\
\hline Volatilidad del subyacente & $\begin{array}{l}\text { Incertidumbre sobre los precios del } \\
\text { proyecto }\end{array}$ \\
\hline Fecha del ejercicio & $\begin{array}{l}\text { Plazo hasta que la oportunidad } \\
\text { desaparece }\end{array}$ \\
\hline Dividendos u otros rendimientos & Mantenimiento de la opción \\
\hline $\begin{array}{l}\text { Su valor depende de la revalorización } \\
\text { esperada del subyacente }\end{array}$ & $\begin{array}{l}\mathrm{Su} \text { valor depende la revalorización } \\
\text { esperada de los flujos de caja }\end{array}$ \\
\hline Tasa de interés libre de riesgo & Tasa de interés libre de riesgo \\
\hline
\end{tabular}

Fuente: Mascareñas (1999)

En ese orden de ideas, el modelo propuesto por Loch \& Bode (2001) en la evaluación de estos proyectos requiere reconocer las amenazas y oportunidades propias de sucesos inciertos y la flexibilidad gerencial como respuesta a ellos; siendo las opciones reales uno de los instrumentos utilizados en la valoración de la flexibilidad gerencial.

Pero, el autor advierte que la debilidad de este último modelo consiste en la dependencia de los precios de las opciones en un activo replicante que reproduzca los beneficios del proyecto en 
todos sus posibles estados; pues estos no lograrían representar o replicar los riesgos inherentes de los proyectos de investigación.

La respuesta de Loch \& Bode (2001) al problema anterior es que,

"En esta situación, un árbol de decisiones es una mejor herramienta para representar las opciones de gestión durante la ejecución del proyecto y evaluar su valor. Un árbol de decisión es equivalente a la fijación de precios de opciones para riesgos que se pueden tasar en los mercados financieros (si se incluye explícitamente la negociación de valores) $\mathrm{y}$, además, puede incorporar riesgos y flexibilidad que no se negocian en los mercados financieros".

Las opciones reales, son aplicadas en la teoría de evaluación financiera para activos no financieros sino activos reales, definen la importancia en la valoración de la flexibilidad dentro de las etapas del proceso de valoración, los cuales posibilitan la vinculación como activos corporativos (Allen, Bhattacharya, Rajan, \& Schoar, 2008). Enuncian que estas pueden ser un terreno cultivable, una patente de explotación de una nueva variedad de planta, o la oportunidad de expansión de una empresa agropecuaria.

Sin embargo, Vasseur (2016), en su investigación incentiva la aplicación de opciones reales a través de equivalentes de certeza y funciones de utilidad como medio alterno en la valuación de las patentes. Para los autores, factores como la incertidumbre, la flexibilidad gerencial en distintas etapas de los proyectos y las especificidades de cada inversión son elementos esenciales para que un modelo de valor pueda brindar una serie de opciones o posibilidades al inversionista (dependiendo de la percepción del riesgo de este). Para ellos,

"El modelo propuesto permite representar las preferencias de inversionistas no diversificados en mercados incompletos y sus diferentes actitudes frente al riesgo. Y proponen aplicar en futuras investigaciones otro tipo de funciones de utilidad con la finalidad de comparar resultados”. (Pareja Vasseur \& Cadavid Pérez, 2016).

Recíprocamente, Brandao et al. (2018), coincide con la idea de multiplicidad de etapas de los proyectos de inversión, indicando que cada fase está sujeta a diversas fuentes de incertidumbre, pero de igual forma, en ellas existen flexibilidades implícitas durante como después del proceso de desarrollo. Sobre este aspecto ellos sentencian que: 
"Una característica importante de esta clase de proyectos es que a medida que la empresa incurre en un costo e invierte, aprende tanto sobre la dificultad de desarrollar e implementar el proyecto como sobre las condiciones del mercado. Desarrollamos un modelo de opciones reales donde la firma actualiza continuamente sus perspectivas de finalización oportuna y las condiciones futuras del mercado. Esta información se puede utilizar para decidir de manera óptima si se justifica o no una mayor inversión, dados los ingresos futuros esperados de toda la empresa" (Brandao et al., 2018)

Bajo un modelo similar al de cartera de opciones, Maier et al. (2020), plantea que "el valor de las carteras de opciones reales a menudo se ve afectado por fuentes de incertidumbre tanto exógenas como endógenas, la mayoría de los enfoques de valoración existentes consideran sólo las primeras y descuidan las segundas”. (Maier et al. 2020)

Frente a este hecho, en su artículo los escritores brindan la orientación necesaria para valorar carteras de opciones reales (abandono, aplazamiento, suspensión de pagos, etc.) dependientes entre sí bajo los tipos de incertidumbre de orden endógeno (costo de finalización de la decisión y valor de rescate) y exógeno (ingresos operativos y tasa de crecimiento).

\section{Fundamentos teóricos de la propuesta metodológica: árboles binomiales.}

El método de árboles binomiales fue planteado por Cox, Ross y Rubinstein y se basa, según sus autores, en operaciones algebraicas elementales que permiten estimar el precio del activo subyacente en tiempo discreto (Cox et al., 1979). El supuesto que se asume para el activo subyacente es que sigue una caminata aleatoria o random walk (Hull, 2005).

\section{Caso de estudio}

Empresa del sector agrícola dedicada a la producción de aguacate, con una extensión de 14.5 hectáreas y 20 asociados, para un promedio de 0.7 ha, por finca productora que desean expandir a 2 hectáreas por cada asociado, \$450.849.978, promedio expansión por hectárea \$17.680.391 costos operativos $51 \%$, cmv $22 \%$, con un nivel de producción y, un porcentaje de deuda del $80 \%$, costo de la deuda de $12.5 \%$, . 


\section{Método de solución}

Se realizará un análisis del marco conceptual de la norma internacional de información financiera, con respecto a la nic 41 , y niif 16 , niif 2 , en donde retomaremos las dinámicas contables y su tratamiento desde la implementación del cultivo hasta su desarrollo y renovación del cultivo.

Entre tanto la metodología que se utiliza para la valoración del caso en mención es la de árboles binomiales, la cual muestra las diferentes vías que puede tomar la opción(financiera o real). Trigeorgis y Tsekrekos (2018) indican que el precio de la acción en el momento actual será $\mathrm{S}_{0}$, donde en el momento $\mathrm{t}+1$ el precio podrá tener dos opciones, uno de crecimiento señalado como $\mathrm{S}_{\mathrm{u}} \mathrm{y}$ otro de decrecimiento $\mathrm{S}_{\mathrm{d}}$, de acuerdo a la siguiente expresión:

$$
s_{u}=S_{0}, u \text { y } S_{d}=S_{0}, d
$$

Donde u señala la medida de expansión y d es la medida de contracción, de igual forma, se debe cumplir que $\mathrm{u}>1$ y $0<\mathrm{d}<1$.

La metodología de árboles binomiales utilizada en el presente caso, se tomó con referencia a lo expresado por Benninga y Tolkowsky (2002), a partir de la siguiente ecuación:

$$
p=\frac{e^{r \Delta t}-d}{u-d}
$$

En la anterior expresión $\mathrm{r}$ es la tasa libre de riesgo, mientras $\Delta \mathrm{t}$ el período en el que se evalúa la opción, de la siguiente manera:

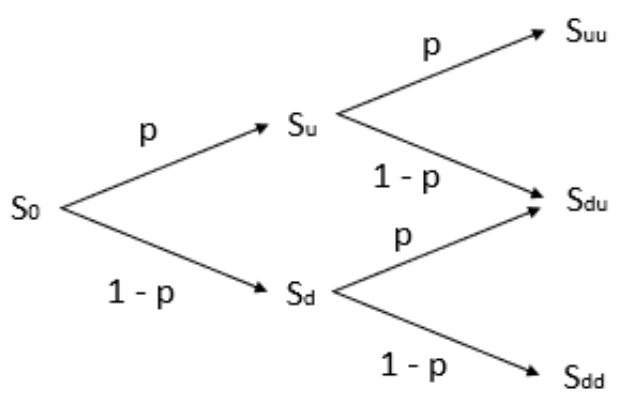

Figura 1. Árbol binomial de dos pasos 


\section{Resultados}

\subsection{Valoración del proyecto actual}

Se valoró la situación actual del proyecto, a partir del valor contable de las 14.5 hectáreas sembradas actualmente, teniendo en cuenta los flujos futuros del mismo para un horizonte de 10 años; se aclara que la situación de los ingresos está definida por el supuesto de vender aguacate hass al mercado nacional al no contemplarse dentro de los costos la certificación en buenas prácticas agrícolas (BPA), que permiten exportarlo a un mayor precio.

Tabla 2. Valoración de línea de activo biológico para 14.5 hectáreas

\begin{tabular}{|c|c|c|c|c|c|c|c|c|c|c|c|c|c|c|c|c|c|c|c|c|c|}
\hline & & 2020 & & 2021 & & 2022 & 2023 & & 2024 & & 2025 & & 2026 & & 2027 & & 2028 & & 2029 & & 2030 \\
\hline Utilidad Operativa & & & $\$$ & 132.859 .135 & $\$$ & 137.550.967 & 142.407.012 & $\$$ & 147..433.020 & $\$$ & 152.634.938 & $\$$ & 157.583.069 & $\$$ & 163.155 .494 & $\$$ & 168.922 .953 & $\$$ & 174.892 .273 & $\$$ & 181.070 .519 \\
\hline Depreciación & & & $\$$ & 1.193 .200 & $\$$ & 1.193 .200 & $1,193.200$ & $\$$ & 1.193 .200 & $\$$ & 1.193 .200 & $\$$ & 1.629 .053 & $\$$ & 1.629 .053 & $\$$ & 1.629 .053 & $\$$ & 1.629 .053 & $\$$ & 1.629 .053 \\
\hline Variación KTNO & & & $-\$$ & 119.486 .466 & $-\$$ & 4.182 .026 & 4.328 .397 &.$\$$ & 4.479.891. & $-\$$ & 4.636 .687 & $-\$$ & 4.798 .971 &.$\$$ & 4.966 .935 & $-\$$ & 5.140 .778 & $-\$$ & 5.320 .705 & $\$$ & 157.340 .858 \\
\hline Inversiones & $-\$$ & 176.775 .692 & & & & & & & & & & & & & & & & & & & \\
\hline Flujo de Caja Operativo & $-\$$ & 176.775.692 & $\$$ & 14.565.869 & $\$$ & 134.562 .140 & 139.271 .815 & $\$$ & 144.146.329 & $\$$ & 149.191.450 & $\$$ & 154.413.151 & $\$$ & 159.817.611 & $\$$ & 165.411 .228 & $\$$ & 171.200.621 & $\$$ & 340.040 .430 \\
\hline Impuestos operativos & $\$$ & - & $\$$ & 42.514 .923 & $\$$ & 44.016.309 & 45.570 .244 & $\$$ & 47.178.566 & $\$$ & 48.843.180 & $\$$ & 50.426 .582 & $\$$ & 52.209 .758 & $\$$ & 54.055.345 & $\$$ & 55.965.527 & $\$$ & 57.942 .566 \\
\hline Flujo de caja libre & $-\$$ & 176.775 .692 & $-\$$ & 27.949 .054 & $\$$ & 90.545 .831 & 93.701 .571 & $\$$ & 96.967 .762 & $\$$ & 100.348 .270 & $\$$ & 103.986.569 & $\$$ & 107.607 .853 & $\$$ & 111.355 .883 & $\$$ & 115.235 .093 & $\$$ & 282.097 .864 \\
\hline Factor de Descuento & & & & 0,877 & & 0,768 & 0,673 & & 0,589 & & 0,516 & & 0,452 & & 0,396 & & 0,347 & & 0,304 & & 0,266 \\
\hline Valor Presente del FCL & $-\$$ & 176.775 .692 & $-\$$ & 24.501 .981 & $\$$ & 69.523 .933 & 63.015 .085 & $\$$ & 57.115.872 & $\$$ & 51.769.159 & $\$$ & 46.986 .192 & $\$$ & 42.586 .186 & $\$$ & 38.598 .438 & $\$$ & 34.984.293 & $\$$ & 75.010 .132 \\
\hline VPN & $\$$ & 278.311.616 & & & & & VPNMedio & $\$$ & 140.671.519 & & & & & & & & & & & & \\
\hline TIR & & $35,61 \%$ & & & & & TIR Medio & & $26,48 \%$ & & & & & & & & & & & & \\
\hline
\end{tabular}

Cifras expresadas en pesos cop.

Fuente: Elaboración propia, 2020.

De esta manera, en la tabla 1 se observa que el VPN del proyecto en la situación actual fue de \$278.311.611, el cual se convierte en el indicador base para comparar el proyecto de la ampliación.

9.2 Valoración del proyecto de ampliación.

La evaluación de la ampliación del área sembrada en aguacate hass contempla la inversión necesaria para lograr la exportación de al menos el 40\% de la producción de las fincas, lo cual posibilita cotizar el valor de la fruta al 90\% de la TRM COP/USD; de esta manera la tasa de descuento a trabajar para la valoración del proyecto de ampliación se construyó teniendo en cuenta los parámetros dados por Damodaran, teniendo en cuenta el $\mathrm{Kd}$ del $12.5 \%$ que 
corresponde a la tasa de interés más baja en el mercado para créditos de aguacate, establecida por Finagro (Tabla 1)

De esta manera, el VPN del proyecto fue de $\$ 580.948 .000$, con una TIR del $27.81 \%$ (Tabla 2.)

Tabla 3. VPN y TIR del proyecto de ampliación en la siembra

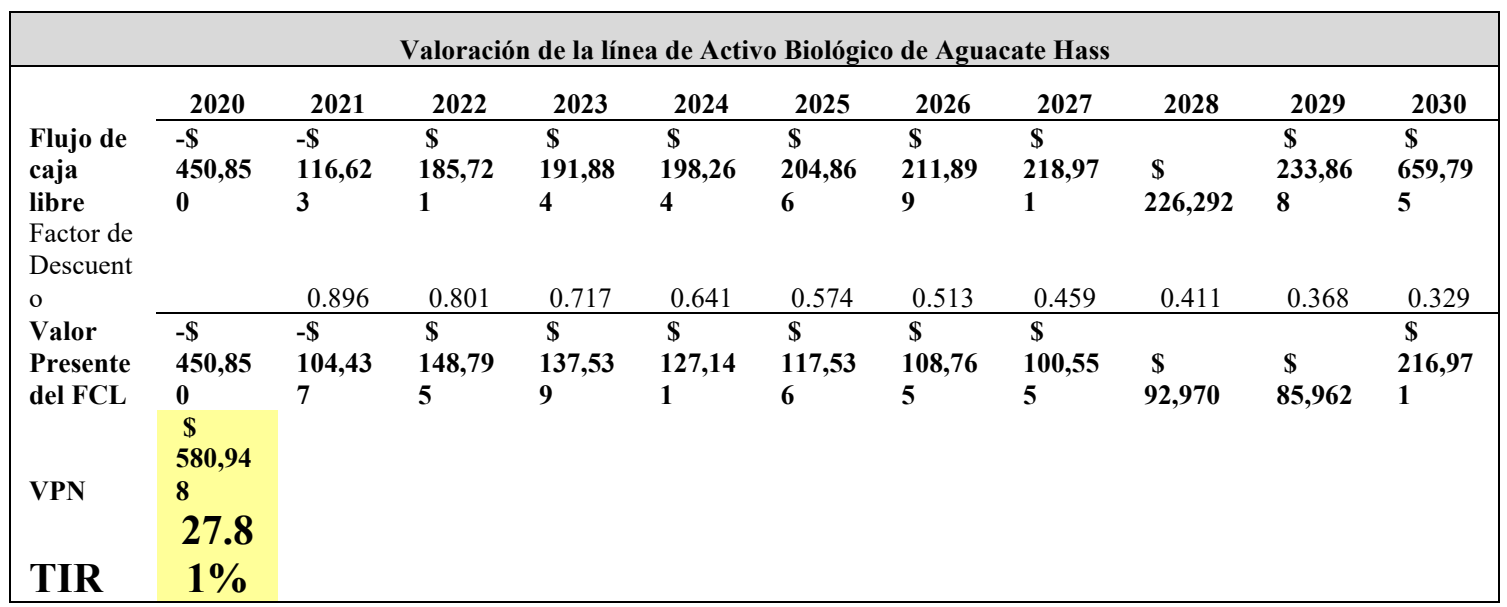

Cifras en millones de pesos

Elaboración propia 2020.

Como resultado de la valoración por el método tradicional con el VPN, se obtiene un valor de $\$ 580.947 .842$. cop, es un valor representativo, pero se debe tener en cuenta que no es el adecuado porque su TIR está por debajo del importe estipulado por la asociación, lo anterior obedece al tiempo y la inversión. Teniendo en cuenta que se estipulo una tir de $30 \%$, el proyecto no sería aceptado.

9.2.1 Volatilidad y riesgo del proyecto de ampliación.

Para definir los rubros más representativos del proyecto, se usó el análisis de tornado, arrojando que los rubros que más influencian el proyecto fueron los costos operativos, la mano de obra y la inversión para la expansión, entre otros, tal como se muestra en la Figura 1. 
Año 14 / Núm. 35 / Enero-junio 2021

Figura 1 Análisis de tornado de flujo de fondos

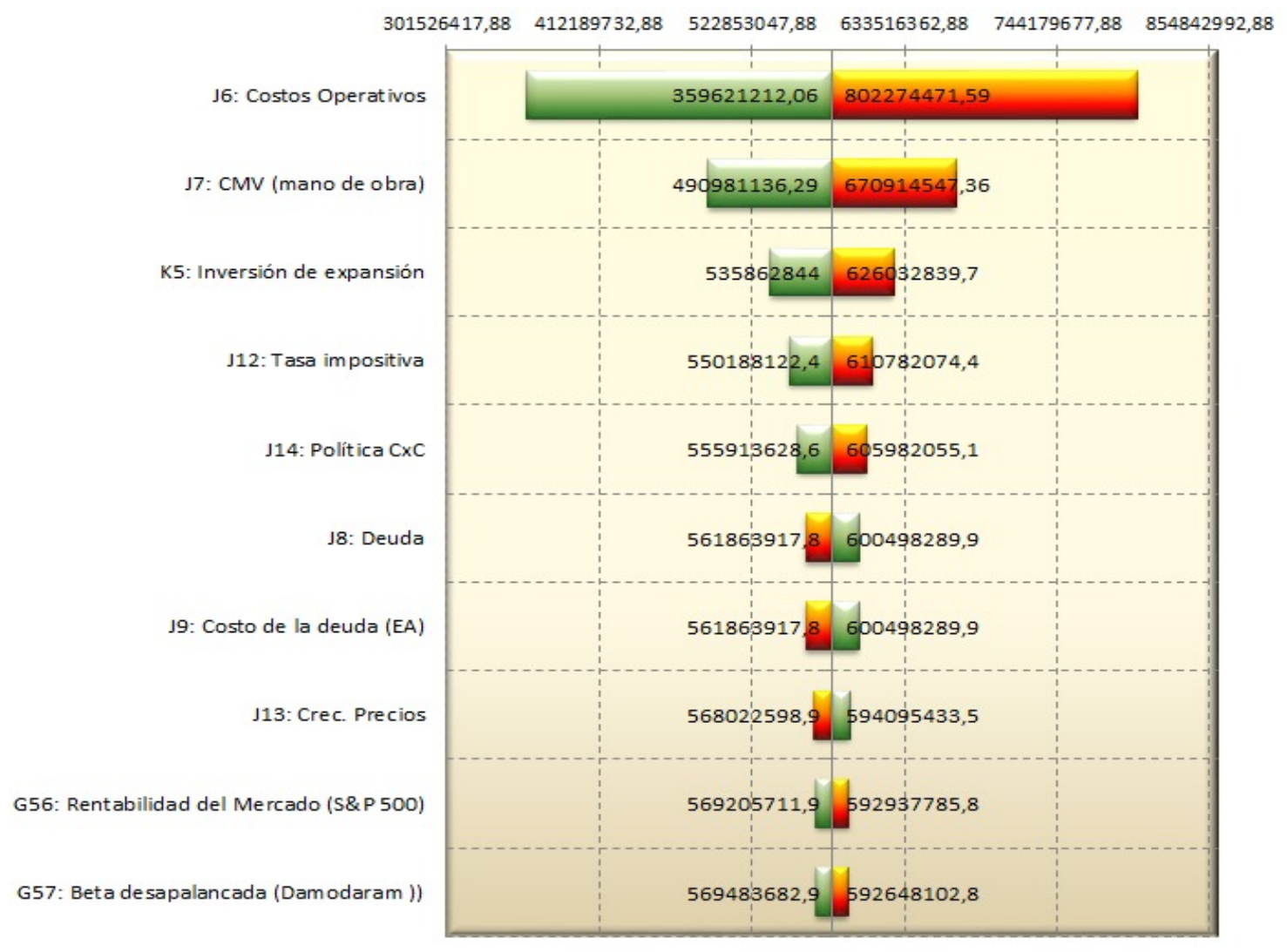

Fuente: salida del @Risk Simulator.

Después de hallar la distribución de probabilidad más ajustada a los datos anteriores, a un WACC del 12.5\%, correspondiente a la tasa de interés más baja en el mercado para el aguacate, (Tabla 1); posteriormente se simularon 10.000 iteraciones, arrojando una desviación estándar del $10.37 \%$ (figura 2). 
Figura 2. Volatilidad del flujo de caja del proyecto de ampliación del cultivo

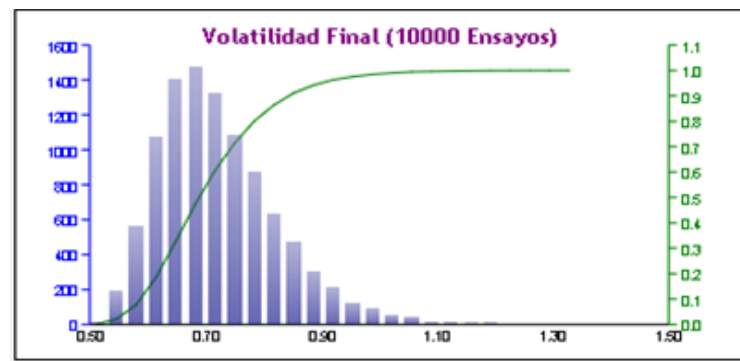

Tipo Doble vínculo

\begin{tabular}{|l|r|}
\hline Estadisticas & Resultado \\
\hline Número de Pruebas & 10000 \\
Media & 0.7023 \\
Mediana & 0.6854 \\
Desviación Estándar & 0.1037 \\
Variación & 0.0107 \\
Coeficiente de Variación & 0.1476 \\
Máximo & 1.3252 \\
Mínimo & 0.4749 \\
Rango & 0.8503 \\
Asimetría & 0.9731 \\
Curtósis & 1.4742 \\
25\% Percentil & 0.6272 \\
$75 \%$ Percentil & 0.7608 \\
Precisión de Error al 95\% de Confianza & $0.2893 \%$ \\
\hline
\end{tabular}

Fuente: salida del@Risk Simulator.

\subsubsection{Construcción de los árboles binomiales}

Para hallar el valor del proyecto por opciones reales, es necesario iniciar hallar primero el árbol del subyacente, el árbol de contracción, por expansión y por último el árbol de la opción, teniendo en cuenta los siguientes parámetros:

Figura 3 Parámetros de entrada

\begin{tabular}{|l|r|}
\hline Volatilidad & $10.37 \%$ \\
\hline Precio inicial del activo & $1,031,797,820$ \\
\hline U & 1.11 \\
\hline$d$ & 0.9 \\
\hline$q$ & 54.48 \\
\hline $1-q$ & 45.52 \\
\hline Contracción & $83 \%$ \\
\hline Ingreso por abandono & $479,589,289$ \\
\hline Expansión & $53 \%$ \\
\hline Costo de expansión & $307,588,309$ \\
\hline Iasa libre de riesgo & $1,47 \%$ \\
\hline
\end{tabular}

Fuente: elaboración propia 
Árbol del subyacente:

Los resultados arrojados por el árbol del subyacente, son tomados a partir del valor presente del flujo de caja en este caso de \$1.031.797.820 con el valor de u, en donde da como resultado positivo el importe 1 . De igual forma para encontrar el valor negativo se procede a multiplicar el flujo de caja por el valor de $\mathrm{d}$, dicho procedimiento se repite hasta completar con todos los momentos del árbol.

Figura 4. Árbol del Subyacente

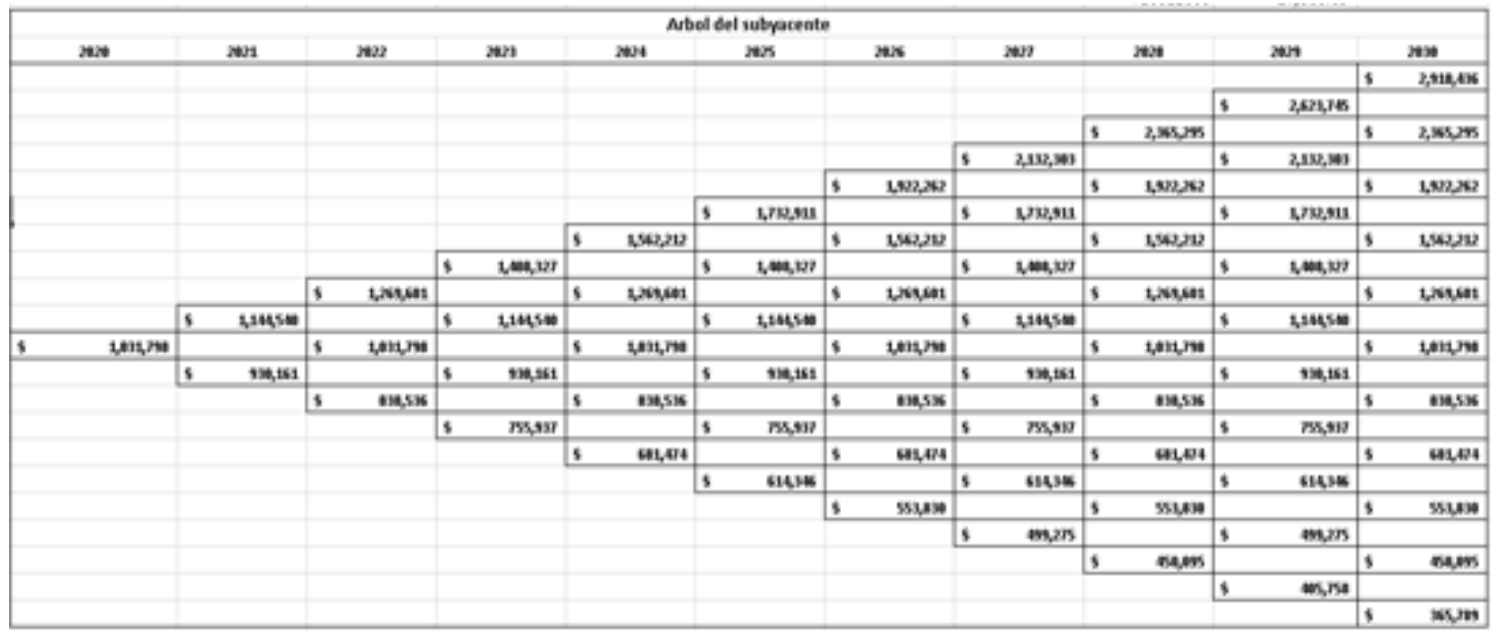

Cifras: en millones de cop.

Fuente: Elaboración propia, 2020.

9.2.3.Arbol del ejercicio por contracción:

Después de haber calculado el árbol del subyacente se debe realizar el mismo procedimiento para hallar el árbol por contracción, el cual tiene características especiales en cuanto a que para el cálculo de este se tomó como base el ingreso futuro del actual proyecto para los próximos 10 años, como medida de comparación en el caso de que no se hiciera nada, y correspondiente al 83\% del VPN del proyecto de la ampliación del área, el cual se calculó con la siguiente expresión:

Max((Ingreso por contracción - Valor Presente del Flujo de caja libre *\%contracción;0) 
Figura 5. Árbol por contracción

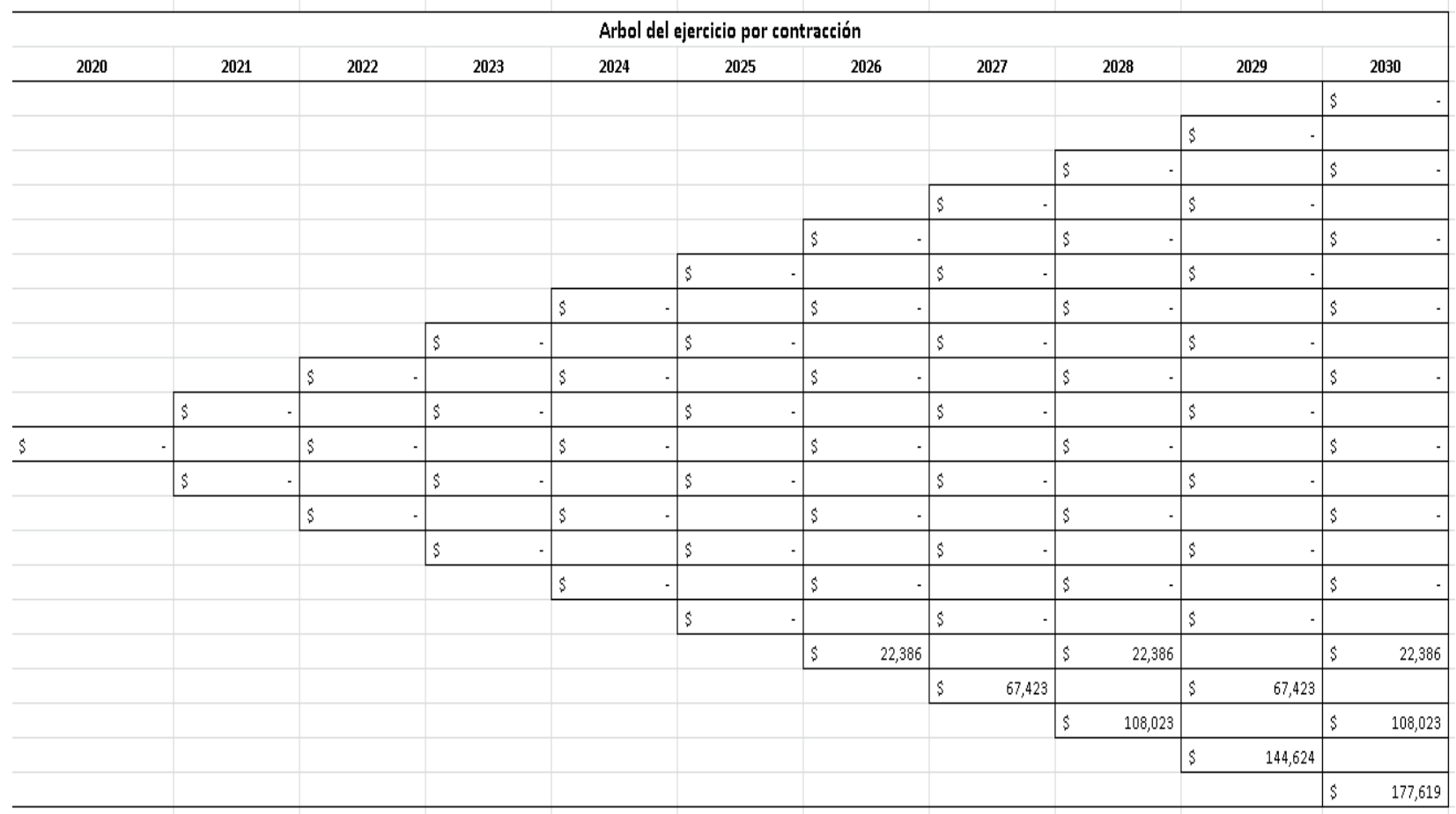

Cifras expresadas en millones de pesos cop.

Fuente: Elaboración propia, 2020.

9.2.4 Árbol del ejercicio por expansión:

De igual manera se construyó el árbol para el escenario de la expansión del área teniendo en cuenta el costo de la misma a incurrir partiendo de un valor $\$ 307.588 .309$, lo cual representaría un incremento en los flujos de caja del 53\%, claramente se puede definir como una opción call americana, determinada por la siguiente expresión:

Max((Valor Presente del Flujo de caja libre*\% expansión - Valor de expansión;0) 
Figura 6. Árbol por Expansión

\begin{tabular}{|c|c|c|c|c|c|c|c|c|c|c|c|}
\hline \multicolumn{12}{|c|}{ Arbol del ejercicio por expansión } \\
\hline \multirow[t]{11}{*}{2020} & 2021 & 2022 & 2023 & 2024 & 2025 & 2026 & 2027 & 2028 & 2029 & & 2030 \\
\hline & & & & & & & & & & $\$$ & $1,233,369$ \\
\hline & & & & & & & & & $1,081,578$ & & \\
\hline & & & & & & & & 944,739 & & $\$$ & 944,739 \\
\hline & & & & & & & 821,380 & & 821,380 & & \\
\hline & & & & & & 710,172 & & 710,172 & & $\$$ & 710,172 \\
\hline & & & & & 609,918 & & 609,918 & & 609,918 & & \\
\hline & & & & \begin{tabular}{|l|l}
519,540 \\
\end{tabular} & & \begin{tabular}{|l|l}
519,540 \\
\end{tabular} & & 519,540 & & $\$$ & 519,540 \\
\hline & & & 438,064 & & 438,064 & & 438,064 & & 438,064 & & \\
\hline & & 364,614 & & 364,614 & & 364,614 & & 364,614 & & $\$$ & 364,614 \\
\hline & 298,399 & & 298,399 & & 298,399 & & 298,399 & & 298,399 & & \\
\hline \multirow[t]{11}{*}{238,707} & & 238,707 & & 238,707 & & 238,707 & & 238,707 & & $\$$ & 238,707 \\
\hline & 184,894 & & 184,894 & & 184,894 & & 184,894 & & 184,894 & & \\
\hline & & 136,383 & & 136,383 & & 136,383 & & 136,383 & & $\$$ & 136,383 \\
\hline & & & 92,650 & & 92,650 & & 92,650 & & 92,650 & & \\
\hline & & & & 53,224 & & 53,224 & & 53,224 & & $\$$ & 53,224 \\
\hline & & & & & 17,683 & & 17,683 & & 17,683 & & \\
\hline & & & & & & $\$$ & & $\$$ & & $\$$ & \\
\hline & & & & & & & $\$$ & & $\$$ & & \\
\hline & & & & & & & & $\$$ & & $\$$ & \\
\hline & & & & & & & & & $\$$ & & \\
\hline & & & & & & & & & & $\$$ & \\
\hline
\end{tabular}

\section{Cifras en millones COP.}

Fuente: Elaboración propia, 2020.

Una vez hecho los análisis independientemente se procede a analizar de manera simultánea los escenarios, a través del árbol de la opción. 
Figura 7: Árbol de la opción simultanea

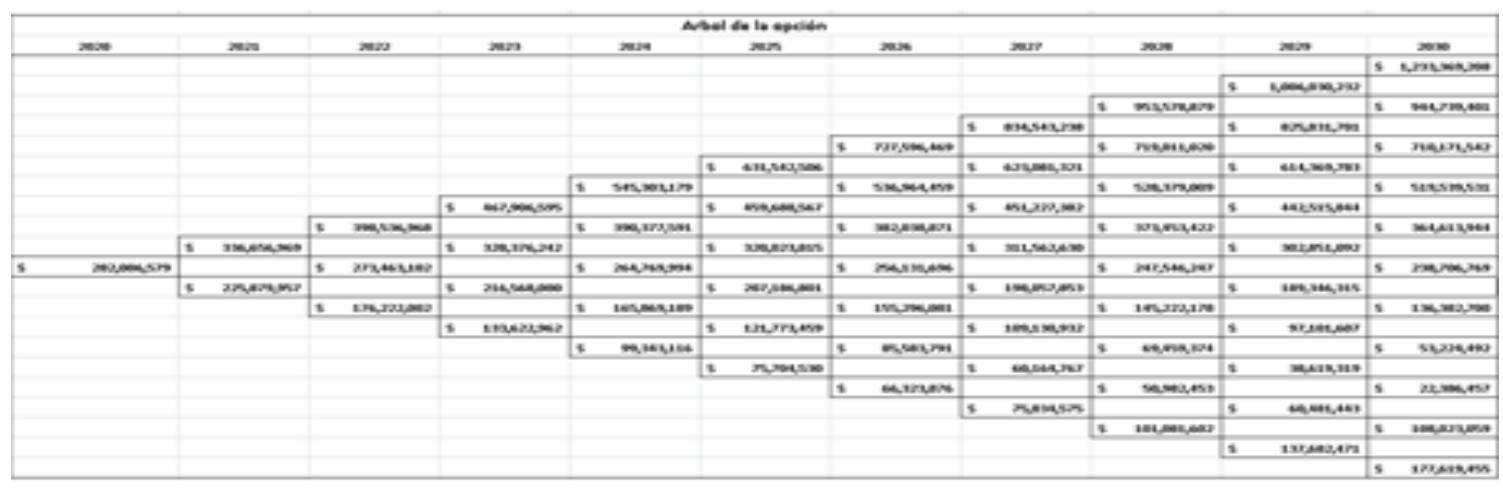

Cifras en millones COP.

Fuente: Elaboración propia, 2020.

De esta forma, el resultado obtenido corresponde a una opción simultánea, que demuestra el valor de ambas opciones y la flexibilidad del proyecto, esencial para la toma de decisiones de la gerencia, que corresponde a contraer, expandir o mantener el nivel de operaciones del proyecto en un momento determinado.

En consecuencia, el valor total del proyecto bajo la metodología de árboles binomiales, está definido por la sumatoria del Valor presente neto más el valor de las opciones reales, tomado este como el árbol binomial para el primer año de evaluación del proyecto, de la siguiente manera:

\begin{tabular}{|l|l|c|c|c|}
\hline $\begin{array}{c}\text { Valor Total } \\
\text { Proyecto }\end{array}$ & $=$ & VPN & + & $\begin{array}{c}\text { Valor Opciones } \\
\text { Reales }\end{array}$ \\
\hline 863.034 .421 & $=$ & $\$ 580.947 .842$ & + & $\$ 282.086 .579$ \\
\hline
\end{tabular}


Lo anterior indica que aunque el proyecto genera rentabilidad con la metodología tradicional no es lo suficientemente atractivo para los inversionistas por tanto se hizo necesario la valoración por opciones reales la cual incluyó la flexibilidad de actuar ante distintos escenarios que ofrece esta metodología.

\section{Conclusiones}

El método tradicional de valoración de proyecto, fue menor que el de valoración de opciones reales, debido a que no se incluye la incertidumbre dentro de las diferentes etapas del proyecto.

Al utilizar la metodología bajo la valoración de opciones reales en los proyectos de inversión, se puede identificar la flexibilidad, como valor agregado a los inversionistas.

El proyecto de inversión, al realizar el comparativo con la metodología tradicional, cumple con el indicador de la Tir, estando por encima de la utilidad deseada por los inversionistas.

Los supuesto con que construyó el método de valoración de opciones reales facilitan la gestión del riesgo del proyecto en cuanto se analizan distintos escenarios donde se puede tomar decisiones de abandono, expansión o mantener la inversión, generando información relevante en momento dado del proyecto.

\section{Referencias}

Allen, F., Bhattacharya, S., Rajan, R., \& Schoar, A. (2008). The contributions of Stewart Myers to the theory and practice of corporate finance. Journal of Applied Corporate Finance, 20(4), 819.

Brandao, L. E., Fernandes, G., \& Dyer, J. S. (2018). Valuing multistage investment projects in the pharmaceutical industry. European Journal of Operational Research, 271(2), 720-732. doi:10.1016/j.ejor.2018.05.044 
Carrillo Moncayo, D. A. (2015). Determinación de costos de producción y la fijación del precio de venta en el sector avícola del cantón Píllaro (Bachelor's thesis, Universidad Técnica de Ambato. Facultad de Contabilidad y Auditoría. Carrera de Contabilidad y Auditoría.)

Cox, J. C., Ross, S. A., \& Rubinstein, M. (1979). Option pricing: A simplified approach. Journal of financial Economics, 7(3), 229-263.

Departamento Administrativo Nacional de Estadística, DANE. (15 de Noviembre de 2019). Sistema de información de precios SIPSA. Recuperado el 19 de Noviembre de 2019, de Sistema de información de precios SIPSA: https://www.dane.gov.co/index.php/estadisticas-portema/agropecuario/sistema-de-informacion-de-precios-sipsa

De Reyck, B., Degraeve, Z., \& Gustafsson, J. (2004). Valuing real options in incomplete markets. London Business School, http://www. sal. tkk. fi/publications/pdf-files/mrey04. pdf, downloaded, 12(19), 2010.

Dixit, A., \& Pindyck, R. (1994). Investment under Uncertainty. Princeton, NJ, EEUU: Princeton University Press.

García Rivera, K., López Acosta, M. L., \& Cerón Espinosa, H. (2015). Efecto de la NIC 41 en la actividad agrícola en Colombia.

Hull, J. C. (2018). Options, Futures and other Derivates (10 ed.). New York: Pearson.

Loch, C., \& Bode-Greuel, K. (2001). Evaluating growth options as sources of value for pharmaceutical research projects. $R$ and D Management, 31(2), 231-248.

Maier, S., Pflug, G., \& Polak, J. (2020). Valuing portfolios of interdependent real options under exogenous and endogenous uncertainties. European Journal of Operational Research, 285(1), 133-147. doi:https://doi.org/10.1016/j.ejor.2019.01.055

Myers (1977). Determinants of Corpo-rate Borrowing. Journal of Financial Economics, 5(2). 147-175. doi.org/10.1016/0304-405X(77)90015-0

Myers, S. C., \& Turnbull, S. M. (1977). Capital budgeting and the capital asset pricing model: Good news and bad news. The Journal of Finance, 32(2), 321-333. 
Palavecinos, B. S. (2011). Valor Razonable: un modelo de valoración incorporado en las Normas Internacionales de Información Financiera. Estudios Gerenciales, 27(118), 97-114.

Smith, J. E., \& Nau, R. F. (1995). Valuing risky projects: Option pricing theory and decision analysis. Management science, 41(5), 795-816.

Tohmatsu, D. T. (2013). In the Dark: What Many Boards and Exceutive Still Don't Know About The Health of Their Businesses.

Uribe M. L. (2011). Enfoque a las normas internacionales de contabilidad en Colombia NIIFs(1ra Ed.). Bogotá, ed. Nueva Legislación Ltda

Vasseur, J. P., \& Pérez, C. C. (2016). Valoración de patentes farmacéuticas a través de opciones reales: equivalentes de certeza y función de utilidad. Contaduría y administración, 61(4), 794814.

\section{CÓMO CITAR}

Fabio Lopez, H., Colmenares Florez, R., \& Lara Papamija, D. (2021). Valoración de activos biológicos aplicados en el cultivo de Aguacate Hass, a través de la evaluación por expansión bajo la metodología de opciones reales. Revista De Investigación Académica Sin Frontera: División De Ciencias Económicas Y Sociales, (35).

https://doi.org/10.46589/rdiasf.vi35.408

\section{ČRIS - UNISON}

$=\ldots$ Sistema de Gestión de la Investigación
- Dialnet

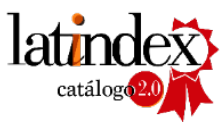
IIf IJIFACTOR
REDIB

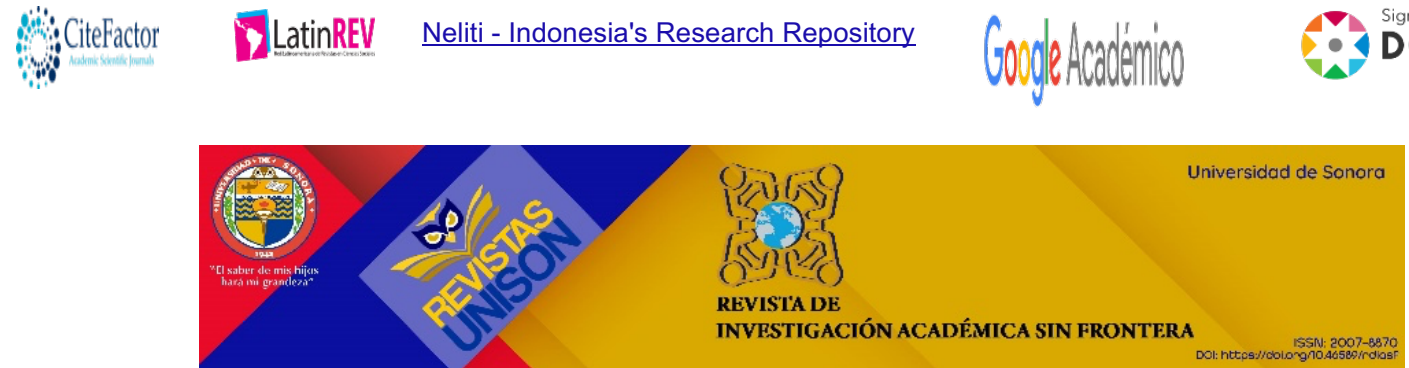

